\title{
Compreensão de leitura em alunos de sétima e oitava séries do ensino fundamental
}

\author{
Compreensão em leitura
}

\author{
Katya Luciane de Oliveira \\ Evely Boruchovitch \\ Acácia Aparecida Angeli dos Santos
}

\section{Resumo}

\begin{abstract}
A leitura está presente em todos os momentos na escola. Há diversas situações durante o dia que requerem o uso dessa habilidade. Explorar as diferenças na compreensão em leitura, considerando o gênero, tipo de escola e série escolar, de estudantes das $7^{\mathrm{a}}$ e $8^{\mathrm{a}}$ séries do ensino fundamental foi o objetivo desta pesquisa. Participaram 206 estudantes matriculados nas $7^{\mathrm{a}}$ e $8^{\mathrm{a}}$ séries do ensino fundamental de escolas públicas e particulares. A compreensão textual foi medida por meio da técnica de Cloze. Os resultados indicaram diferença estatisticamente significativa na leitura dos estudantes em razão do gênero, do tipo de escola (pública e privada) e da série escolar.
\end{abstract}

Palavras-chave: Leitura; habilidade em leitura; Teste de Cloze.

\section{Text comprehension in $7^{\text {th }}$ and $8^{\text {th }}$ grade basic school students}

\begin{abstract}
Reading is present in all school moments. There are a lot of situations during the day, which requires this skill. The objective of this research was to explore the reading comprehension differences in relation to gender, type of school and school grade level. The sample was composed of 206 students from public and private high schools attending the $7^{\text {th }}$ and $8^{\text {th }}$ grades. Reading comprehension was measured using a text in accordance with Cloze's technique. The results showed significant differences in student's reading comprehension in reason of gender, type of school (public and private) and school grade level.

Keywords: Reading; reading skill; Cloze Testing.
\end{abstract}

\section{Comprensión de lectura en alumnos del séptimo y octavo grado de la enseñanza primaria}

\section{Resumen}

La lectura está presente en todos los momentos en la escuela. Hay varias situaciones durante el día que requieren el uso de esta habilidad. Explorar las diferencias en la comprensión en lectura, considerando el sexo, tipo de escuela y grado escolar, de estudiantes del $7^{\circ}$ y $8^{\circ}$ grado de la enseñanza primaria fue el objetivo de esta investigación. Participaron 206 estudiantes inscriptos en el $7^{\circ}$ y $8^{\circ}$ grado de la enseñanza primaria de escuelas públicas y particulares. La comprensión textual fue medida por la técnica de Cloze. Los resultados indicaron diferencia estadísticamente significativa en la lectura de los estudiantes en razón del sexo, del tipo de escuela (pública y privada) y del grado escolar.

Palabras clave: lectura, habilidad en lectura, test de Cloze. 


\section{Introdução}

A leitura permite não só o exercício do poder individual de análise como também da tomada de decisão e possibilita um entendimento mais amplo da realidade, exigindo um aprendizado contínuo. Pode-se dizer que quanto mais se lê e se compreende, maior será o entendimento dos fatos e a compreensão do mundo. A escola e, em especial, o ensino fundamental ainda são os principais cenários nos quais se desenvolve a competência para a leitura (Salvia \& Ysseldyke, 199I; Ellis, 1995; Soares, 1995).

Um aspecto preocupante, no entanto, tem sido apontado em publicações especializadas que denunciam o crescimento do fracasso escolar no ensino fundamental. De um lado, os professores buscam explicações sobre o não aprendizado do aluno, atribuindo a culpa pelo baixo rendimento a características do próprio aluno e de seu ambiente (Fini \& Calsa, 2006). Do outro lado, os estudantes apresentando dificuldades de aprendizagem e se consideram incapazes, pouco inteligentes ou com problemas de outra natureza (Sisto \& Martinelli, 2006). Independentemente do caráter das explicações, a dificuldade de leitura aparece como uma das principais causas dos insucessos do aluno no ensino fundamental, havendo poucos estudos com foco na busca de alternativas para a questão (Ferreira \& Dias, 2002; Santos, 2004).

$\mathrm{Na}$ direção de identificar mais objetivamente aspectos que podem ser melhorados, Boruchovitch (200I) avaliou alunos de $3^{\mathrm{a}}, 5^{\mathrm{a}}$ e $7^{\mathrm{a}}$ séries do ensino público fundamental e seus resultados indicaram que os estudantes apresentam dificuldades de compreensão em leitura e conhecem poucos recursos adequados para melhorar ou aprimorar essa habilidade. A autora destaca ainda que o desenvolvimento de um repertório mais diversificado de estratégias para a compreensão em leitura poderia ser um dos caminhos para a superação dos problemas verificados.

Constata-se então, a necessidade do diagnóstico da leitura, no ensino fundamental, principalmente pelo fato de que a causa mais freqüente dos encaminhamentos psicopedagógicos é a dificuldade em leitura. No entanto, sem que se identifiquem as dificuldades específicas, a força de medidas interventivas de remediação são pouco viáveis (Salvia \& Ysseldyke, 1991).

O foco central para discutir o diagnóstico em leitura está na definição de bons ou maus leitores. De uma forma geral, segundo Kopke Filho (200I), adotase o critério de que um bom leitor é aquele que consegue decodificar as palavras, utiliza a contextualização para imprimir significado ao texto, bem como apresenta velocidade na leitura.

O papel da cognição na compreensão em leitura é discutido por Kintsch e van Dijk (1978). A memória e a aprendizagem são elementos constituintes da compreensão, de modo que a proficiência em leitura ocorre quando o leitor é capaz de transportar a nova informação para novos contextos. Nesse contexto, vale relembrar o princípio de Matthew que sugere que as crianças que tiveram um bom começo na aprendizagem em leitura apresentarão uma melhor proficiência (Nicholson, 1999).

Carriedo e Alonso-Tapia (1995) e Santos e Santos (2002) destacam que é possível recorrer ao uso adequado de estratégias cognitivas e metacognitivas para favorecer o entendimento do texto. Valendo-se de estratégias adequadas, o leitor poderá desenvolver suas habilidades em leitura, entendendo o texto lido e abstraindo as idéias mais relevantes. Contudo, a compreensão somente se concretiza quando o pequeno leitor consegue utilizar conhecimentos já aprendidos para compreender essa nova informação (Otto, 1992; Santos, 2004).

Assim, como observa Nicholson (1999), o leitor proficiente utiliza meta-compreensão à medida que apresenta a habilidade para refletir sobre seu próprio processo cognitivo, percebendo quando não entendeu algo (conhecimento sobre compreensão). Ele demonstra, também, habilidade de regular seu próprio pensamento enquanto lê, fazendo analogias e críticas ao conteúdo lido (conhecimento de como compreender).

Um recurso muito utilizado para mensurar a proficiência na compreensão em leitura é o teste de Cloze. O instrumento baseia-se na técnica criada por Taylor (1953), no qual um texto de aproximadamente 250 palavras é preparado, omitindo-se todos os $5^{\circ} \mathrm{s}$ vocábulos e no lugar da palavra omitida é colocado um 
traço proporcional ao tamanho da palavra excluída para que o estudante possa incluí-la.

Para Condemarin e Milicic (1988), o Cloze é um recurso interativo entre leitor e o texto uma vez que possibilita ao leitor conhecer suas próprias habilidades de compreensão e facilita a esse realizar uma discriminação entre a compreensão escrita e a compreensão fonológica das palavras impressas do texto. À medida que esses processos interagem e encontram um ponto de equilíbrio, o leitor obtém uma compreensão profunda do texto lido visto que toma consciência da estrutura interna deste, o que amplia sua sensibilidade, resultando na compreensão da leitura que, por sua vez, apresenta níveis de dificuldade.

Embora haja predominância no Brasil de pesquisas com a técnica de Cloze com estudantes universitários (Santos, 1990; Santos, 1991; Centofanti, Ferreira \& Del Tedesco, 1997; Santos, Primi, Taxa \& Vendramini, 2002; Oliveira, Santos \& Primi, 2003; Oliveira, Suehiro \& Santos, 2004; Oliveira \& Santos, 2005; dentre outros), também é possível recuperarse na literatura estudos que utilizam a técnica com estudantes do ensino fundamental. Algumas dessas pesquisas são apresentadas nos trabalhos de Braga (198I), Bitar (1989), Zucolloto (200I), Oliveira, Boruchovitch e Santos (2006), demonstrando que o interesse pela técnica não é recente e tem sido mantido ao longo dos anos.

Um dos primeiros estudos realizados no país com - Cloze foi o de Molina (1979). Em sua investigação com 1399 estudantes do ensino fundamental e médio a autora avaliou a inteligibilidade de textos. Para tanto, quinze textos de livros didáticos utilizados na época foram selecionados e preparados de acordo com a técnica de Cloze. Os resultados evidenciaram a relação entre desempenho no teste e o grau de escolaridade dos estudantes.

Em pesquisa mais recente, Santos (2004) utilizou - Cloze como técnica de diagnóstico e de desenvolvimento da compreensão em leitura. $O$ estudo foi desenvolvido com 24 alunos de $5^{\mathrm{a}}$ série, com média de idade de II,8 anos de uma escola pública do interior paulista. A coleta de dados ocorreu em situação de sala de aula e envolveu a aplicação de pré e pós- teste e um programa de intervenção com o uso do Cloze gradual em textos retirados de livros didáticos, apropriados para o nível de escolaridade dos participantes. A comparação dos resultados do pré e póstestes apontaram para uma diferença significativa, demonstrando que o Cloze era um instrumento adequado, tanto para a avaliação como para o desenvolvimento da compreensão em leitura.

Visando a aumentar os conhecimentos relativos à compreensão em leitura, mensurada por meio da técnica de Cloze, o presente trabalho teve como objetivo comparar o nível de compreensão em leitura de estudantes do ensino fundamental, considerando-se as variáveis sexo, tipo de escola e série escolar. Pretendeu-se ainda averiguar a diferença entre o desempenho dos alunos em razão da autopercepção do seu desempenho escolar, já que, em linhas gerais, estudos evidenciam relações diferentes entre esta variável e o desempenho acadêmico (Shell, Brunning \& Colvin, 1995; Mayer, 1998).

\section{Método}

\section{Participantes}

O estudo foi realizado com 206 alunos matriculados nas $7^{\mathrm{a}}(42,2 \% ; n=87)$ e $8^{\mathrm{a}}(57,8 \% ; n=119)$ séries do ensino fundamental de escolas públicas $(34,5 \% ; n=7 I)$ e particulares $(65,5 \% ; n=135)$ do sul de Minas Gerais o gênero feminino representou $5 \mathrm{I}, 5 \%(n=107)$ da amostra e o masculino $48,5 \%$ $(n=99)$. A média de idade foi de 14 anos e 10 meses $(D P=0,9)$, sendo a idade máxima de 18 anos e a mínima de 13 anos.

\section{Instrumento}

Um texto preparado de acordo com a técnica de Cloze em sua versão tradicional foi utilizado e todos os $5^{\circ} \mathrm{s}$ vocábulos do texto foram omitidos. No local da omissão foi colocado um traço de tamanho proporcional à palavra excluída. $O$ estudante deveria preencher o espaço com a palavra que ele entendia ser adequada ao contexto. $O$ texto continha aproximadamente 250 vocábulos e 40 omissões, sendo adotada a forma de correção literal. 
Vale ressaltar que $\circ$ texto foi especialmente desenvolvido para avaliar a compreensão da leitura em alunos do ensino fundamental, tendo sido construído por Santos (2005) para utilização com essa população. A prova possui evidências de validade visto que apresentava evidência de validade de critério, distinguindo alunos de $5^{\mathrm{a}}$ a $8^{\mathrm{a}}$ séries. A pontuação é dada, considerando-se zero para erros ou espaços em branco e um ponto, para cada acerto. Havia ainda uma questão fechada do tipo Likert que abordava como o aluno se auto-avaliava em relação ao seu desempenho durante aquele semestre, correspondendo às categorias 'Muito bem', 'Bem', 'Regular', 'Mal' e 'Muito mal'.

\section{Procedimento}

A aplicação ocorreu de forma coletiva em horário previamente agendado em cada instituição de ensino. Os pais dos alunos ou responsáveis legais assinaram o termo de consentimento livre e esclarecido. Após um breve rapport, os participantes foram informados acerca dos objetivos da pesquisa, bem como o fato da atividade não ser considerada para a nota da disciplina. De uma forma geral, a aplicação durou aproximadamente I5 minutos em cada sala de aula Em todas as ocasiões, os professores, que estavam ministrando alguma disciplina no momento da aplicação, permaneceram no local acompanhando a atividade. Cabe ainda destacar que os cuidados éticos tomados na presente pesquisa foram baseados e estão em consonância com a Resolução 196/96 do Conselho Nacional de Saúde.

\section{Resultados}

Os dados foram organizados em planilhas e submetidos à estatística descritiva e inferencial, conforme o objetivo deste estudo. A Tabela I mostra a autopercepção de desempenho escolar dos estudantes.

Observa-se que a maior parte da amostra (83,9\%; $n=173$ ) apresentou uma autopercepção predominantemente positiva acerca do próprio desempenho escolar. Poucos $(16,1 \% ; n=33)$ foram aqueles que ti-
Tabela I. Distribuição das freqüências e porcentagens do desempenho escolar percebido $(N=206)$.

\begin{tabular}{lrc}
\hline \multirow{2}{*}{ Categorias } & \multicolumn{2}{c}{ Número de estudantes } \\
\cline { 2 - 3 } & $\mathrm{F}$ & $\%$ \\
\hline Muito bem & 46 & 22,3 \\
Bem & 127 & 61,6 \\
Regular & 26 & 12,7 \\
Mal & 4 & 1,9 \\
Muito mal & 3 & 1,5 \\
\hline
\end{tabular}

veram uma autopercepção negativa. No que se refere à compreensão em leitura, a média de acertos da amostra total $(N=206)$ no teste de Cloze foi de 23,9 $(D P=5,2)$ e o número de acerto mínimo foi de $I$ e o máximo de 35 .

Para avaliar se havia diferença no desempenho no Cloze e na autopercepção do próprio desempenho foi utilizada a Análise de Variância. Para tanto, os alunos foram divididos em três grupos. O primeiro grupo foi composto por estudantes que avaliaram o próprio desempenho como 'muito bom' $(22,3 \% ; n=46)$, no segundo grupo encontravam-se aqueles que se classificaram na categoria 'bom' $(6 \mid, 6 \% ; n=127)$ e o terceiro grupo foi composto por estudantes com avaliações negativas ( $16,1 \% ; n=33)$. Os resultados indicaram não haver diferença significativa entre os grupos considerando-se $[F=(2,20)=0, \mid 3 ; p=0,87)]$. As médias dos grupos foram $M=23,8$ para $\circ$ primeiro grupo, $M=24$, I para o segundo e $M=23,3$ para $\circ$ terceiro.

A diferença de desempenho no Cloze entre os gêneros foi observada pelo teste $t$ de Student. Os dados mostraram que o gênero feminino apresentou uma média mais alta de acertos no Cloze $(M=25)$ em relação ao gênero masculino $(M=22,8)$, diferença essa, estatisticamente significativa $(t=3,04 ; p=0,00)$.

Para verificar se havia diferença entre a pontuação no Cloze, o tipo de escola (pública e privada) e a série escolar ( $7^{\mathrm{a}}$ e $8^{\mathrm{a}}$ séries), recorreu-se também ao teste $t$ de Student. Os dados evidenciaram que houve diferença estatisticamente significativa entre o número de acertos e o tipo de escola $(t=7,92 ; p=0,00)$. A Figura I apresenta as médias de acertos dos alunos nas respectivas escolas. 


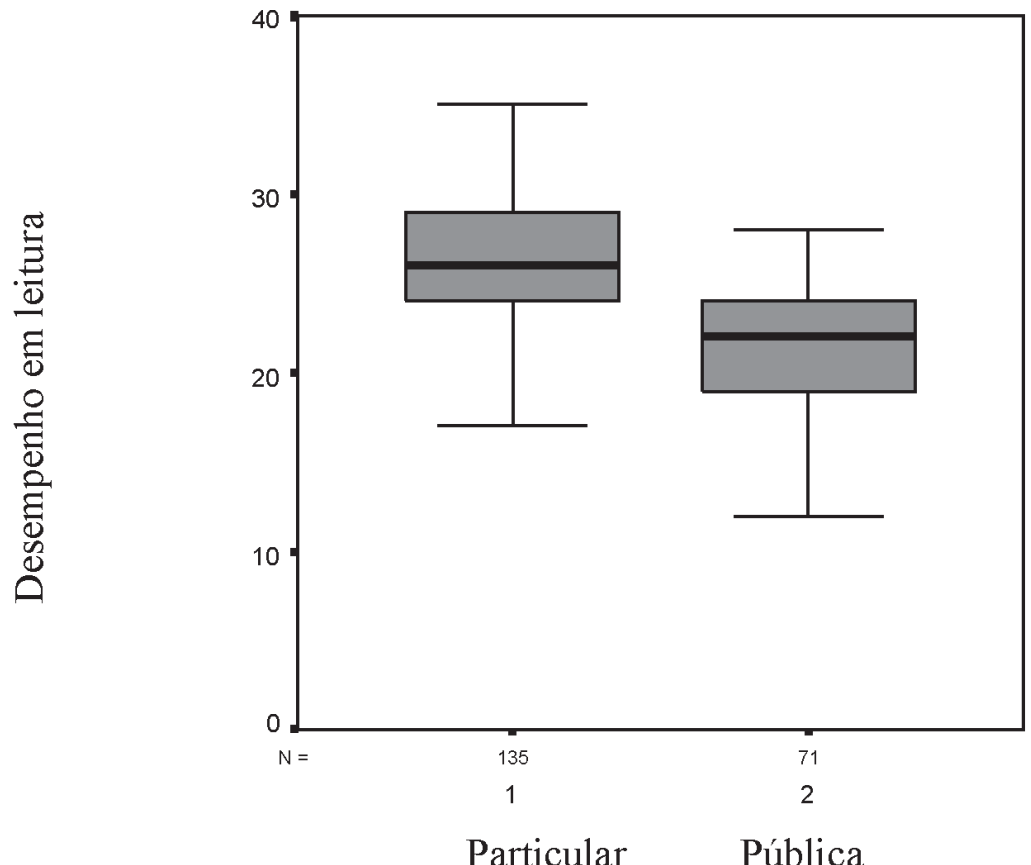

Tipo de escola

Particular Pública

Figura I. Boxplot dos acertos obtidos pelos alunos considerando o tipo de escola $(N=206)$.

Observou-se que a média de acertos dos alunos matriculados na escola particular (grupo I; $n=135$ ) foi maior do que na escola pública (grupo $2 ; n=71$ ). $\mathrm{Na}$ escola pública houve um número maior de lacunas no teste de Cloze que não foram preenchidas.

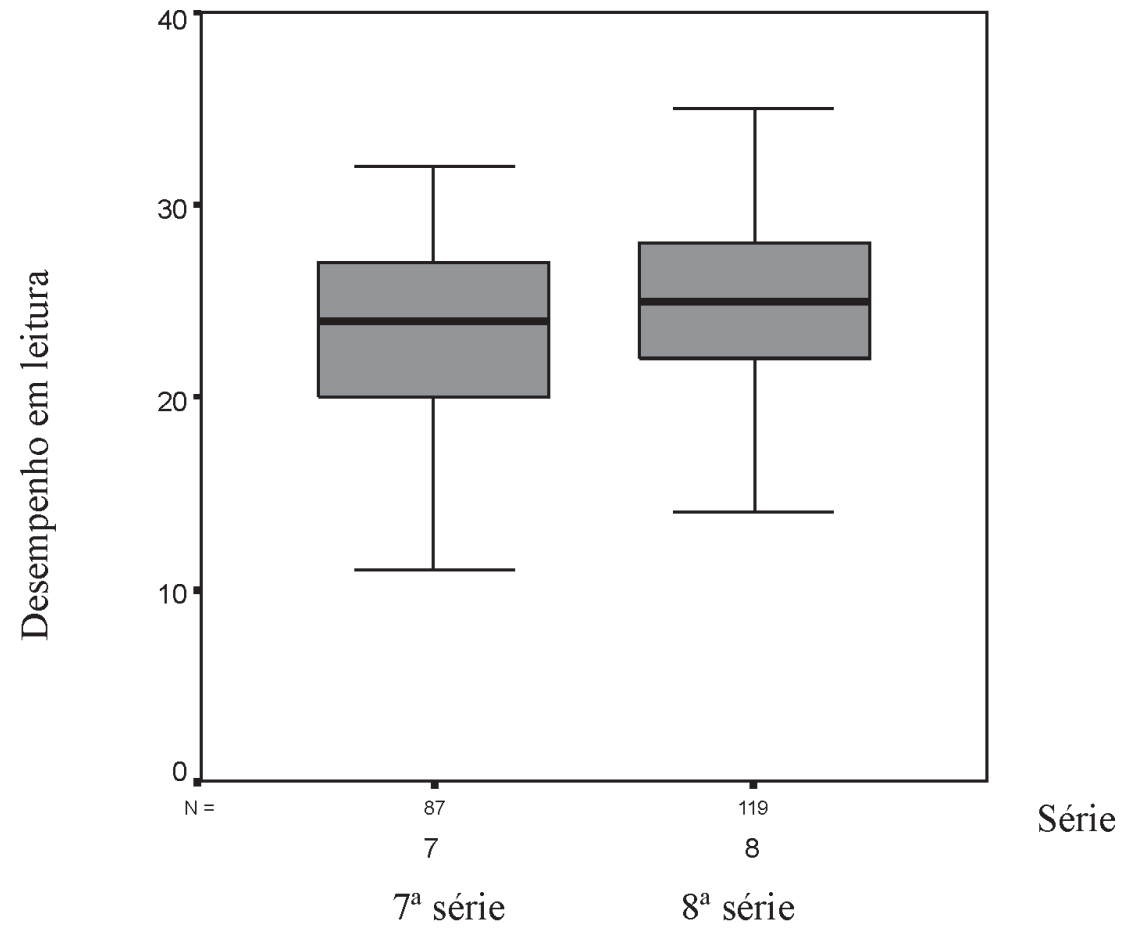

Figura 2. Boxplot dos acertos obtidos pelos alunos considerando a série escolar $(N=206)$. 
Constatou-se, como era de se esperar, que os alunos matriculados na série mais avançada ( $8^{\mathrm{a}}$ série; $n=119)$ apresentaram um melhor desempenho na compreensão em leitura do que os que estavam em um nível escolar abaixo ( $7^{a}$ série; $n=87$ ).

\section{Discussão}

Em linhas gerais, o presente estudo permitiu constatar que o desempenho dos alunos no teste de Cloze foi um pouco superior à metade possível de acertos nesse instrumento. Melhores resultados emergiram entre os participantes mais velhos, da série mais avançada provenientes de escolas particulares e do gênero feminino. A maioria da amostra apresentou uma autopercepção de desempenho positiva. Entretanto, esta variável não se associou siginificativamente ao desempenho dos participantes no Cloze.

Observou-se também que a média de acertos dos alunos da escola pública $(M=20,45)$ quando comparados aos da escola particular $(M=25,85)$, foi abaixo do desejável para leitores de nível independente que é de $57 \%$ de acertos (Santos, 2005). É importante lembrar que só a compreensão em níveis mais elevados de abstração é que contribui para o desenvolvimento do pensamento crítico (Salvia \& Ysseldyke, 199I; Ellis, 1995; Kopke Filho, 200I). Os resultados obtidos no presente estudo permitem a suposição de que esse conjunto de habilidades está comprometido nos estudantes da amostra, especialmente nos advindos da escola pública.

Referente ao fato das meninas da amostra terem tido uma melhor compreensão do texto do que os meninos verificou-se que dados similares foram encontrados por Oliveira, Boruchovitch e Santos (2005a). Entretanto, estudos sobre a compreensão em leitura e sua relação com a variável sexo no ensino fundamental ainda são inespecíficas e escassas, o que dificulta um maior aprofundamento da discussão. Aponta-se, pois, a necessidade de que pesquisas futuras investiguem melhor o papel dessa variável na compreensão em leitura.

A análise por tipo de escola revelou que os alunos da escola privada obtiveram um melhor desempe- nho no Cloze do que aqueles da escola pública. As escolas privadas, de uma forma geral, apresentam diversos estímulos que favorecem a leitura em seus alunos. Em contrapartida, as instituições públicas atribuem a leitura um caráter de dever ou obrigação e não como o caminho de acesso ao conhecimento, diversão e lazer (Soares, 1995).

Uma hipótese que se poderia aventar sobre o baixo desempenho no Cloze dos alunos da escola pública, do presente estudo, é que a leitura, na escola, de um modo geral, reflete uma visão de um mundo socialmente privilegiado. Essa visão difere, em diversos aspectos, da realidade da maioria dos alunos que estudam na escola pública, que são estudantes economicamente menos favorecidos que, em muitos casos, apresentam deficiências de necessidades básicas como boa alimentação, moradia adequada, roupas, entre outras. Uma pesquisa realizada por Boruchovitch (200I) mostrou que estudantes provenientes das escolas públicas possuíam um repertório pouco diversificado de estratégias de aprendizagem relativas à compreensão da leitura, porém seu estudo se limitou à escola pública. Nota-se que também são escassas as pesquisas nacionais sobre o impacto da variável tipo de escola na compreensão em leitura. Recomenda-se que futuros estudos sejam realizados com amostras maiores para ver se as diferenças encontradas no presente estudo se confirmariam.

Os resultados relativos às variáveis: idade e série escolar foram na direção esperada e confirmam achados de estudos anteriores. Dados similares já foram encontrados em estudos com crianças dessa faixa etária (Braga, I98I; Zucoloto, 200I; Santos, 2005).

Tal como na pesquisa de Shell, Brunning e Colvin (1995) não foram encontradas relações significativas entre os diferentes níveis de autopercepção de desempenho e o desempenho em leitura. Foi interessante notar que os estudantes, embora não tenham apresentado uma boa compreensão textual, mostraram possuir uma boa autopercepção acerca de seu próprio desempenho. Poucos foram aqueles que se auto-avaliaram negativamente como estudantes.

Por um lado, alguns autores mencionam que alunos de diferentes níveis de rendimento acadêmico têm dificuldade de emitir autojulgamentos precisos sobre 
o seu desempenho e tendem a se superestimar, tornando-se mais precisos com o avançar da idade e da escolaridade (Shell, Brunning \& Colvin, 1995; Schunk \& Pajares, 2004). Sob essa perspectiva, é possível aventar a hipótese de que os participantes do presente estudo não tenham a real percepção de suas dificuldades escolares visto que a proficiência em leitura é essencial para o sucesso na maioria das disciplinas do currículo, durante a escolarização formal. Por outro lado, é importante salientar que, no presente estudo, a pergunta se referia ao desempenho geral do estudante naquele semestre letivo e não ao desempenho especificamente na compreensão em leitura. Investigações, que ampliem o exame das relações entre a autopercepção de desempenho em tarefas específicas de leitura, o desempenho escolar propriamente dito e o desempenho em medidas de compreensão em leitura devem fazer parte da agenda da pesquisa nessa área.

\section{Considerações Finais}

Os dados parecem indicar que estudantes de escolas privadas são expostos a uma educação de melhor qualidade, o que, certamente, faz a diferença no desempenho dos mesmos no que diz respeito à compreensão em leitura. Dificuldades em ler e compreender um texto também são resultantes de uma escolarização pobre e, na maior parte das vezes, determinada por condições desfavoráveis a que os alunos foram submetidos ao longo dos anos. Entretanto, mesmo os alunos da escola privada, no presente estudo não obtiveram resultados satisfatórios no teste de Cloze quanto se esperava. Assim, pode-se supor que as escolas estejam priorizando uma leitura mais imediatista, negligenciando o papel essencial que esta tem no desenvolvimento e aprofundamento reflexivo dos fatos para a formação completa do cidadão.

As relações entre o fracasso escolar e a compreensão em leitura são aspectos que devem ter especial atenção de estudos posteriores. A falta de compreensão em leitura pode gerar déficits de aprendizagem em diversos conteúdos específicos. Por conseguinte, a criança que apresenta uma história de defa- sagens na aprendizagem em razão de uma baixa habilidade de compreensão textual pode inevitavelmente fracassar.

Como a escola e o ensino fundamental são os principais canais nos quais se processam a relação entre leitor e leitura, faz-se necessário que os assuntos tratados nos livros didáticos utilizados sejam sensíveis e abordem aspectos da própria realidade e experiência empírica do aluno para que haja uma melhor identificação dele com a leitura. Do contrário, a compreensão é muito dificultada, pois o leitor não consegue associar os conteúdos lidos aos conhecimentos já aprendidos e entender a nova informação (Kintsch \& van Dijk, 1978; Otto, 1992; Carriedo \& Alonso-Tapia, 1995). É fundamental que os estudantes sejam expostos a estímulos adequados que promovam o interesse pela leitura, desde cedo. Sob esse aspecto, Nicholson (1999) aponta que aqueles que tiverem um bom começo na aprendizagem em leitura, como diversos reforços positivos nesse processo, tenderão a apresentar uma melhor proficiência em leitura.

Espera-se que este estudo tenha contribuído para a ampliação dos conhecimentos relativos à compreensão em leitura no ensino fundamental visto que são escassos, principalmente aqueles que pretendem mensurar tal habilidade por meio do teste de Cloze e em relação a variáveis como gênero e tipo de escola e autopercepção de desempenho. Salienta-se, também, que o Cloze tem-se revelado um instrumento eficaz para avaliar a compreensão em leitura dos estudantes, nos diversos segmentos da escolarização.

Observa-se, no entanto, que a amostra se restringiu a duas escolas de uma cidade de Minas Gerais, e baseou-se num único texto de Cloze. Sugere-se, pois que novos estudos sejam realizados com amostras mais diversificadas, com utilização de um número maior de textos nessa técnica e que incluam variáveis contextuais outras como, por exemplo, a família.

Tendo em vista a importância de se desenvolver a competência em leitura, desde o início da escolarização formal, conclui-se pela necessidade de se ultrapassar o caráter remediativo de possíveis intervenções e de se ampliar esforços em direção a propostas de ações preventivas para a formação do leitor crítico, independente e reflexivo (Boruchovitch, 200I; 2004). 


\section{Referências}

Bitar, M. L. (1989). Eficiência dos instrumentos de avaliação em leitura. Dissertação de Mestrado, Pontifícia Universidade Católica de São Paulo, São Paulo.

Boruchovitch, E. (200I). Estratégias de compreensão em leitura de alunos do ensino fundamental. Psicologia Escolar e Educacional, 5, 19-25.

Boruchovitch, E. (2004). A auto-regulação da aprendizagem e a escolarização inicial. Em E. Boruchovitch \& J. A. Bzuneck (Orgs.), Aprendizagem: Processos psicológicos e o contexto social na escola (pp. 55-88). Petrópolis: Vozes.

Braga, S. M. L. (198I). Remediação da leitura: Um estudo com escolares de primeiro grau utilizando a técnica de Cloze. Dissertação de Mestrado, Instituto de Psicologia, Universidade de São Paulo, São Paulo.

Carriedo, N., \& Alonso-Tapia, J. (1995). Comprehension strategy training in content areas. European Journal of Psychology of Education, X(4), 4I I-43I.

Centofanti, E. M., Ferreira, S. M., \& Del Tedesco, T. (I997). Compreensão da leitura por universitários de psicologia. Em G. P. Witter (Org.), Leitura e universidade (pp. 33-60). Campinas: Alínea.

Condemarín, M., \& Milicic, N. (1988). Test de Cloze. Santiago de Chile: Editorial Andrés Bello.

Conselho Nacional de Saúde. Resolução 196/96. Diretrizes e Normas Regulamentadoras de Pesquisa. [citado em 19 de outubro de 2005]. Disponível: http://www.conselho.saude.gov.br

Ellis, A. W. (1995). Leitura, escrita e dislexia: uma análise cognitiva. (2. ed.). (Dayse Batista, Trad.). Porto Alegre: Artmed.

Ferreira, S. P. A., \& Dias, M. G. B. B. (2002). A escola e o ensino da leitura. Psicologia em Estudo, 7, 39-49.

Fini, L. D. T., \& Calsa, G. C. (2006). Matemática e afetividade: alunos desinteresados no ensino fundamental? Em F. F. Sisto \& S. C. Martinelli (Orgs.), A afetividade e dificuldades de aprendizagem (pp. 163-180). Campinas: Vetor.

Kintsch, W., \& van Dijk, T. A. ( 1978). Toward a model of text comprehension and production. Psychological Review, 85, 363-394.

Kopke, F. H. (200I). Estratégias em compreensão da leitura: conhecimento e uso por professores de língua portuguesa. Tese de doutorado em lingüística, Faculdade de Filosofia, Letras e Ciências Humanas, Universidade de São Paulo, São Paulo.
Mayer, R. E. ( 1998). Cognitive, metacognitive and motivational aspects of problem solving. Instructional science, 26, 49-63.

Molina, O. (1979). Avaliação da inteligibilidade de livros didáticos de $I^{\circ}$ e $2^{\circ}$ graus por meio da técnica de Cloze. Tese de Doutorado, Universidade de São Paulo, São Paulo.

Nicholson, T. (1999). Reading comprehension processes. Em G. B. Thompson \& T. Nicholson (Orgs.), Learning to read (pp. I27-I49). Newark: IRA.

Oliveira, K. L., Boruchovitch, E., \& Santos, A. A. A. (2006). Desempenho escolar percebido em alunos da $2^{\mathrm{a}}$ série do ensino fundamental. II Congresso Brasileiro de Psicologia: ciência \& Profissão. Enfrentando as dívidas histórias da sociedade brasileira [On line]. Disponível: www.psicologiacienciaeprofissao.com.br

Oliveira, K. L., \& Santos, A. A. A. (2005). Compreensão em leitura e avaliação da aprendizagem em universitários. Psicologia: Reflexão e Crítica, 18, I I8-124.

Oliveira, K. L., Santos, A. A. A., \& Primi, R. (2003). Estudo das relações entre compreensão em leitura e desempenho em disciplinas na universidade. Revista Interação em Psicologia, 7, 19-25.

Oliveira, K. L., Boruchovitch, E. \& Santos, A. A. A. (2005a). Compreensão da leitura em alunos do ensino fundamental: estudo comparativo por gênero. VII Congresso Brasileiro de Psicologia Escolar e Educacional, [CD-ROM].

Otto, W. (1992). The role of research in reading instruction. Em S. J. Samuels \& A. E. Farstrup (Orgs.), What research has to say about reading instruction (pp. 3-16). Newark: IRA.

Salvia, J., \& Ysseldyke, J. (1991). Avaliação em educação especial e corretiva. São Paulo: Manole.

Santos, A. A. A. (1990). Compreensão em leitura na universidade: um estudo comparativo entre dois procedimentos de treino. Estudos de Psicologia, 7(2), 39-53.

Santos, A. A. A. (1991). Desempenho em leitura: um estudo diagnóstico da compreensão e hábitos de leitura entre universitário. Estudos de Psicologia, 8, 6-19.

Santos, A. A. A. (2004). O Cloze como técnica de diagnóstico e remediação da compreensão em leitura. Interação em Psicologia, 8, 21 7-226.

Santos, A. A. A. (2005). O Cloze na avaliação da compreensão em leitura com alunos de $5^{a} a 8^{a}$ série. Manuscrito não publicado.

Santos, A. A. A., Primi, R., Taxa, F., \& Vendramini, C. M. M. (2002). O teste de Cloze na avaliação da compreensão em leitura. Psicologia: Reflexão e Crítica, 15, 549-560. 
Santos, A. A. A., \& Santos, E. C. P. (2002). Avaliação de um programa de intervenção para o ensino de espanhol. MaracayVE, Paradigma, XXIII(2), 49-76.

Santos, A. A. A., Suehiro, A. C. B., \& Oliveira, K. L. (2004). Habilidades em compreensão da leitura: um estudo com alunos de psicologia. Estudos de Psicologia, 2 I (2), 29-4I.

Schunk, D. H., \& Pajares F. (2004). Self-efficacy in Education revisited. Em D. M. Mclnerney \& S. Van Etten (Eds.), Big Theories Revisited (pp. I I5-I38). Greenwich, Conn: Information Age Publishing.

Shell, D. F., Bruning, R. H., \& Colvin, C. (1995). Self-efficacy, attribution, and outcome expectancy mechanisms in reading and writing achievement: Grade-level and achievement-level differences. Journal educational psychology, 87, 386-398.
Sisto, F. F., \& Martinelli, S. C. (2006). O papel das relações sociais na compreensão do fracasso escolar e das dificuldades de aprendizagem. Em F. F. Sisto \& S. C. Martinelli (Orgs.), A afetividade e dificuldades de aprendizagem (pp. 13-30). Campinas: Vetor.

Soares, M. B. (1995). Comunicação e expressão: o ensino da leitura. Em M. Abreu (Org.), Leituras no Brasil: antologia comemorativa pelo $10^{\circ}$ Cole (pp. 47-7I). Campinas: Mercado das Letras.

Taylor, W. L. (1953). Cloze procedure: a new tool for measuring read-ability. Journalism Quarterly, 30, 4I5-433.

Zucoloto, K. A. (200I). A compreensão da leitura em crianças com dificuldade de aprendizagem na escrita. Dissertação de Mestrado, Universidade Estadual de Campinas, Campinas.

Recebido em: |4/08/2006

Revisado em: 16/11/2006

Aprovado em: 28/02/2007

Sobre as autoras:

Katya Luciane de Oliveira (katya_lincoln@ig.com.br) é Mestre em Psicologia pelo Programa de Pós-graduação Stricto Sensu em Psicologia da Universidade São Francisco. Doutoranda em Psicologia, Desenvolvimento Humano e Educação pela Faculdade de Educação da Unicamp. Docente do curso de Psicologia da Universidade São Francisco.

Evely Boruchovitch (evely@unicamp.br) é Ph.D em Educação pela University of Southern Califórnia, docente da graduação e da pós-graduação do Departamento de Psicologia Educacional da Faculdade de Educação da Unicamp. Bolsista produtividade do CNPq.

Acácia Aparecida Angeli dos Santos (acaciasantos@terra.com.br) é Doutora em Psicologia Escolar e do Desenvolvimento Humano pela USP, docente da graduação no curso de psicologia e no Programa de Pós-graduação Stricto-sensu em Psicologia, da Universidade São Francisco-SP. Bolsista produtividade do CNPq.

Endereço para correspondência:

Katya Luciane de Oliveira

R. Maestro Sebastião Peranovich, 4I5, Atibaia Jardim

12940-000 - Atibaia - SP 